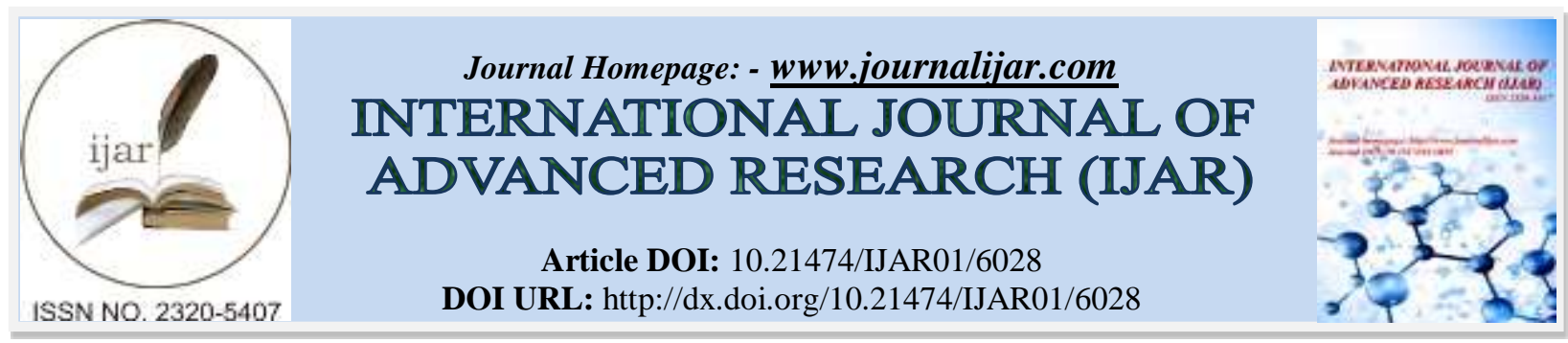

RESEARCH ARTICLE

\title{
AUDIOLOGICAL EVIDENCES ON EFFICACY OF MUCOLITE THERAPY IN PATIENTS WITH SEROUS OTITIS MEDIA.
}

Dr. Prakash. T. K ${ }^{1}$, Dr. Abhilash. $S^{2}$, Dr. Devi. $\mathbf{N}^{3}$ and Dr. Jasjyot Kaur ${ }^{4}$.

1. Reader in ENT, Department Of Otorhinolaryngology. All India Institute Of Speech and Hearing, Mysuru, Karnataka.

2. ENT Surgeon, Department Of Otorhinolaryngology, All India Institute Of Speech and Hearing, Mysuru, Karnataka.

3. Reader in Audiology, Department Of Audiology, All India Institute Of Speech and Hearing, Mysuru, Karnataka.

4. Senior Resident, Department Of Otorhinolaryngology, Northern Railway Central Hospital, New Delhi.

\section{Manuscript Info}

Manuscript History

Received: 11 October 2017

Final Accepted: 13 November 2017

Published: December 2017

Key words:-

Ambroxol, Audiometry, Otitis media,

Audiometric thresholds, Speech audiometry.

\section{Abstract}

Introduction: Serous otitis media (SOM) is defined as collection of fluid in the middle ear cavity behind an intact tympanic membrane without any signs or symptoms of acute inflammation occurred due to Eustachian tube dysfunction. SOM has several potential causes, leading to decreased hearing and learning disabilities. Hence early detection and proper intervention is necessary.

Objective: To compare the audiological findings before and after the mucolite therapy for individuals with serous otitis media.

Materials and Methods: This was a prospective study, conducted in Department of Otorhinolaryngology and Department of Audiology, All India Institute of Speech and Hearing, Manasagangothri, Mysuru from November 2014 to January 2017. 39 patients who were diagnosed as serous otitis media based on history, clinical examination and audiological evaluation are included in the study. Patients were started on mucolite (ambroxol) therapy for 3 months duration. At the end of $1^{\text {st }}$ and $3^{\text {rd }}$ month (Post therapy) audiological testing were repeated.

Results: At the end of $3^{\text {rd }}$ month, the audiometric thresholds were within normal limits. The speech recognition threshold (SRT) and speech identification score (SIS) also revealed a good correlation with the pure tone thresholds.

Conclusion: The learning disability and language problem in school going children, and conductive hearing loss in adults caused by serous otitis media can be effectively treated with mucolite therapy usually, without any surgical intervention.

Copy Right, IJAR, 2017,. All rights reserved.

\section{Introduction:-}

Serous otitis media (SOM) is a pathologic condition of the middle ear in which there is a collection of fluid behind a tympanic membrane without signs or symptoms of acute inflammation. It is one of the commonest otological problems seen in paediatric age group, but in some cases adults may also be affected. The prevalence rate in adults 
is $0.6 \%$. Serous otitis media in children causes hearing loss, otalgia, educational or behavioural problems and speech and language delay. The adult patients presents with a history of blocking sensation in the ear, hearing loss, and mild earache. Such cases need early detection and proper treatment.

The fluid in serous otitis media may be serous or mucoid in nature. The fluid is a mixture of the secretions of the epithelial cells, the goblet cells and the mucus glands along with the inflammatory transudate/exudates which comes through the intracellular spaces from the inflamed submucosa [1]. It is the mucin that is responsible for the variable viscosity of the middle ear fluid. Usage of Ambroxol hydrochloride as an mucolytic agent, decreases the mucus viscosity by altering its structure. In the current study, we examined the effect of oral mucolite therapy (Ambroxol) for 3 months duration in relation with hearing improvement.

Aetiology and Pathophysiology: Eustachian tube dysfunction is the usual cause of serous otitis media. The term 'dysfunction' bears emphasis. It is commonly said that the key etiologic factor is eustachian tube obstruction. More accurately, physiologic dysfunction leads to functional obstruction.

Adenoid hypertrophy and nasopharyngeal carcinoma are possible examples of direct tubal obstruction, although it has been suggested that secondary oedema due to adenoiditis, tonsillitis and sinusitis may also play a role. Heredity seems to be a predominant factor in American Indians, Eskimos and children with Down's syndrome. Eustachian tube dysfunction in children with cleft palate, $75 \%$ of who develop serous otitis media, has a mechanical basis. Recent studies have demonstrated that surfactant can be isolated from the eustachian tube, and there has been, accordingly, speculation as to the possible role of a surfactant deficiency.

Once Eustachian tube dysfunction occurs due to varied etiology, mesotympanic space is not aerated properly, resulting in negative middle ear pressure due to absorption of middle ear gases. When the negative middle ear pressure exceeds the oncotic pressure, a transudate occurs. This transudate causes mucosal irritation and secondary hypertrophy of goblet cells is seen with transformation of pleuripotential basal mucosal cells to a secondary state [2,3]. Serous otitis media has a natural course of spontaneous resolution. Hypertrophied secretory cells degenerate, become inactive, and the effusion resorbs, but the incidence of recurrence is high $[4,5]$.

\section{Materials and Methods:-}

This study was conducted in Department of Otorhinolaryngology and Department of Audiology, from November 2014 to January 2017, after taking informed consent and required clearance from Ethical committee. It is a prospective study. A total number of 39 patients were included in the study. The participants were in the range of 453 years (Mean age: 13.7 years, SD12.1), with 26 males and 13 females. Detailed history and thorough clinical and otoscopic examination with all routine investigations and complete audiological evaluation were done for all the patients.

\section{Inclusion Criteria:-}

Both male and female having Serous otitis media with conductive hearing loss without any sequelae were included as participant for the present study.

\section{Exclusion Criteria:-}

The patients were excluded who had SOM with nasopharyngeal malignancy, with grommet insertion, acute otitis media, adenoid hypertrophy, Down syndrome, any space occupying lesion in the nasopharynx and cleft palate. If the patients are included as participants in any other clinical trial and with previous otologic surgery were also excluded.

\section{Procedure:-}

The study that was conducted is classified as three conditions:

Condition 1 (Pre-treatment): All the participants were assessed for their audiometric thresholds before the treatment with mucolite therapy. Pure tone audiometry (PTA) was carried out using GSI 61 clinical audiometer for air conduction and bone conduction thresholds for frequencies between $250 \mathrm{~Hz}$ to $8 \mathrm{kHz}$ from $250 \mathrm{~Hz}$ to $4 \mathrm{kHz}$ respectively using Modified Hughson and Westlake procedure [6]. Speech audiometry which includes speech recognition thresholds (SRT), and speech identification scores (SIS) was also measured. Immitt ance audiometry was carried out using GSI Tympstar to test ear pathologies. The obtained thresholds and valued were documented. 
After the initial evaluation, the participants were prescribed $1.5 \mathrm{mg} / \mathrm{kg} / \mathrm{BW} /$ day of mucolite (Ambroxol) therapy in divided doses.

\section{Condition 2 (Post treatment, 1 month):-}

All the participants who had been treated for the serous otitis media with mucolite therapy were followed up for the re-evaluation of audiological testing after a period of one month. The air condition and bone condition audiometric thresholds and the speech audiometry that were obtained before the treatment for serous otitis media were again reevaluated and documented. The status of the middle ear pathology was also recorded.

\section{Condition 3 (Post treatment, 3 months):-}

Again after the period of three months the participants were again followed up for the re-evaluation of audiological testing. The air condition and bone condition audiometric thresholds and speech audiometry were again re-evaluated and documented. The status of the middle ear pathology was also recorded. All the testing was carried out in a sound treated room [7]. Prior to the testing, a written consent was obtained from all the participants for their willingness to participate in the study.

\section{Results:-}

Appropriate statistical analyses were performed using the Statistical Package for Social Sciences (SPSS) software version 17 (SPSS Inc. Chicago). Following this, Shapiro-Wilk test of normality was administered. As the normality was not observed, non-parametric test was used. Descriptive statistics (mean, median \& standard deviation) were analyzed for the measurements of pure tone average (PTA), speech recognition threshold (SRT), speech identification scores (SIS) and frequencies of 500Hz, $1 \mathrm{kHz}, 2 \mathrm{kHz}, 4 \mathrm{kHz}, 6 \mathrm{kHz}$ and $8 \mathrm{kHz}$ across both the conditions of pre mucolite treatment (condition 1) and post mucolite treatment after 1 (condition 2) and 3 months (condition 3). The analysis was also carried for the ear canal volume of the participants.

Figure 1:- The mean and standard deviation of pure tone average, speech recognition threshold, speech

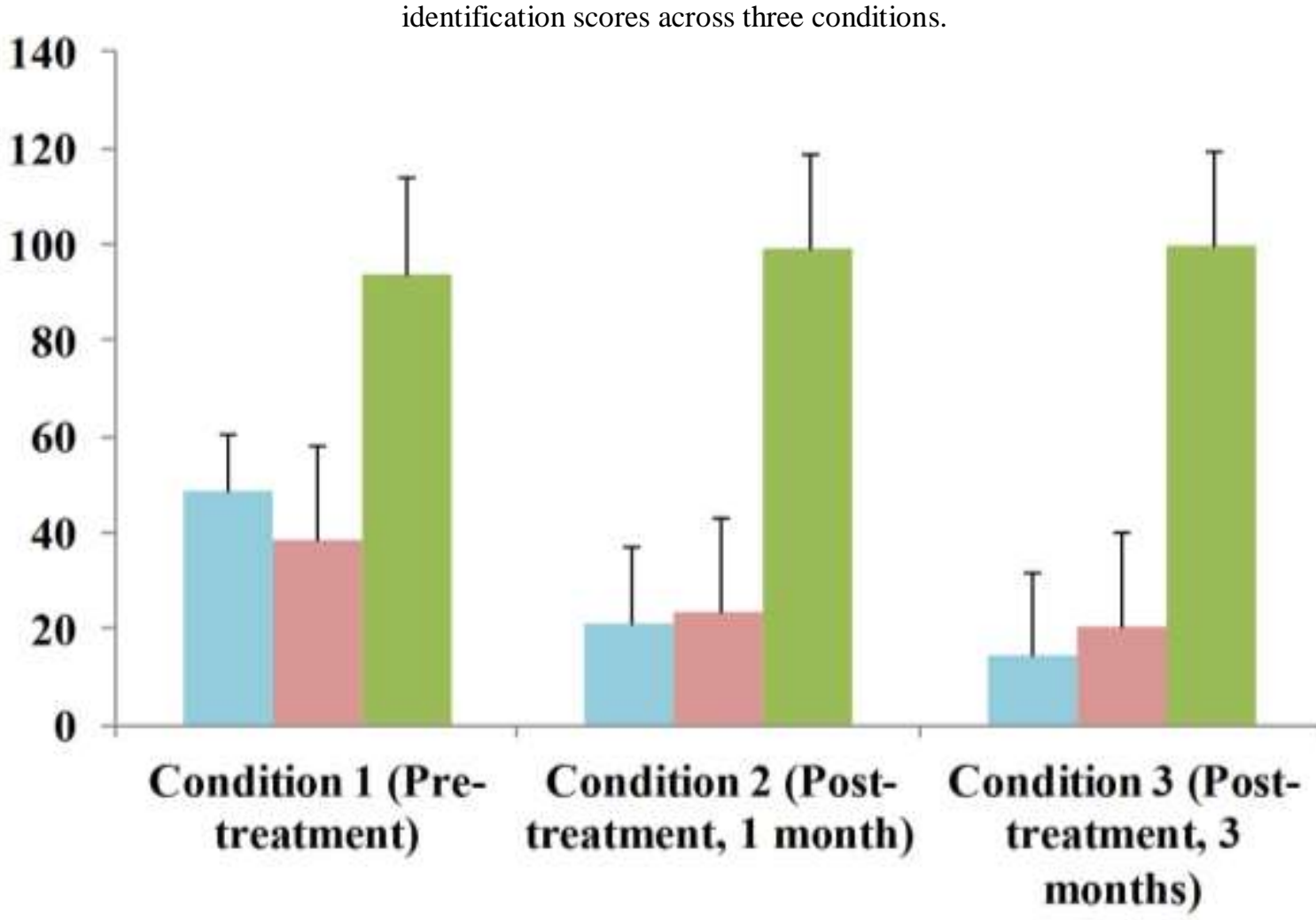

\section{PTA (dB) $=$ SRT (dB) $\quad$ SIS (\%)}


The data from figure 1 reveals that there was difference in PTA, SRT and SIS for all three conditions. The thresholds and scores are better and improved in condition 3 that is the post treatment of mucolite compared to pre treatment and after one month of treatment.

Figure 2:- The mean and standard deviation of air condition thresholds at $250 \mathrm{~Hz}, 500 \mathrm{~Hz}, 1 \mathrm{kHz}, 2 \mathrm{kHz}, 4 \mathrm{kHz}$, and

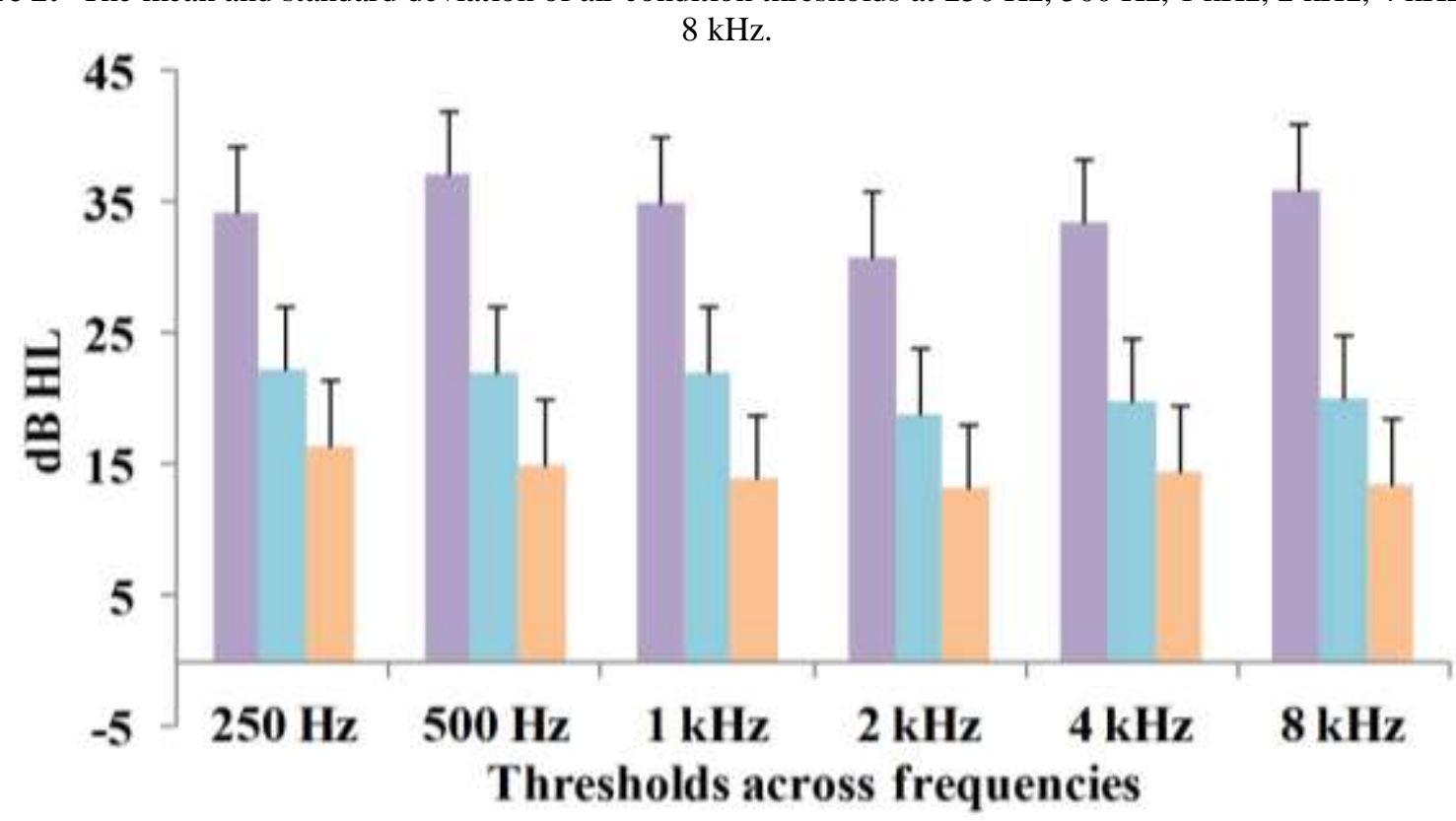

Condition 1 (Pre-treatment)

Condition 2 (Post-treatment, 1 month)

Condition 3 (Post-treatment, 3 months)

The data from figure 2 revels that there was difference in air condition thresholds for all the test frequencies. The thresholds are better and improved in condition 3 that is the post treatment of mucolite compared to pre treatment and after one month of treatment. The thresholds are reported to be improved more at the higher frequencies than the lower frequencies in the hearing range.

Further a non-parametric Friedman test of differences among repeated measures were conducted for all the three conditions. The results of the Friedman test of differences across all the three conditions are reported in Table 1.

Table 1:- Results of Friedman test of differences across all the three conditions.

\begin{tabular}{|l|l|l|}
\hline Parameters & Chi-square Value & Significance P value \\
\hline PTA & 59.92 & 0.000 \\
\hline SRT & 36.23 & 0.000 \\
\hline SIS & 15.1 & 0.001 \\
\hline $250 \mathrm{~Hz}$ & 28.94 & 0.000 \\
\hline $500 \mathrm{~Hz}$ & 55.67 & 0.000 \\
\hline $1 \mathrm{kHz}$ & 52.97 & 0.000 \\
\hline $2 \mathrm{kHz}$ & 45.97 & 0.000 \\
\hline $4 \mathrm{kHz}$ & 49.94 & 0.000 \\
\hline $8 \mathrm{kHz}$ & 27.29 & 0.000 \\
\hline ECV & 9.276 & 0.01 \\
\hline
\end{tabular}

The results of Friedman test of differences reveals that there was significant difference across all the conditions for various parameter of test that was carried out. However, the differences were not found for bone condition 
thresholds. Hence the air bone gap which is the difference between the air condition threshold and bone condition thresholds of the participants were analyzed. The results revealed a significant difference across all the test frequencies of air bone gap across all the conditions. Further the Wilcoxon Signed Ranks Test analysis was carried out for pair wise comparison between condition 1 and 2, condition 1 and 3 , and condition 3 and 2 . The results are summarized in table 2 .

Table 2:- Results of Wilcoxon Signed Ranks Test across each condition

\begin{tabular}{|l|l|l|l|}
\hline Parameters & $\begin{array}{l}\text { Condition 1 \& } \\
\text { Chi-square } \\
\text { (p value) }\end{array}$ & $\begin{array}{l}\text { Condition 3 \& 1 } \\
\text { Chi-square } \\
\text { (p value) }\end{array}$ & $\begin{array}{l}\text { Condition } 3 \& 2 \\
\text { Chi-square } \\
\text { (p value) }\end{array}$ \\
\hline PTA & $-4.93^{*}$ & $-5.01^{*}$ & $-4.71^{*}$ \\
\hline SRT & $-4.614^{*}$ & $-4.557^{*}$ & $-1.882(0.060)$ \\
\hline SIS & $-2.701^{*}$ & $-3.421^{*}$ & -2.058 \\
\hline ECV & $-.544(.586)$ & $-1.629(.103)$ & $-2.200(.068)$ \\
\hline
\end{tabular}

Note: $*$ indicates $\mathrm{p}<0.05$

From table 2 it can be inferred that except for the SRT condition 3 and 2 and all the conditions of ear canal volume there was significant difference at all the conditions across all the parameters that were evaluated. The air bone gap data was also analyzed across each condition, which revealed significant difference.

Figure 3:- The details of audiogram, speech audiometry and immitance sample of a patient with serous otitis media. NOTE: C1 - Condition 1 (Pre-treatment), C2 - Condition 2 (Post treatment, 1 month), C3- Condition 3 (Post treatment, 3 months)

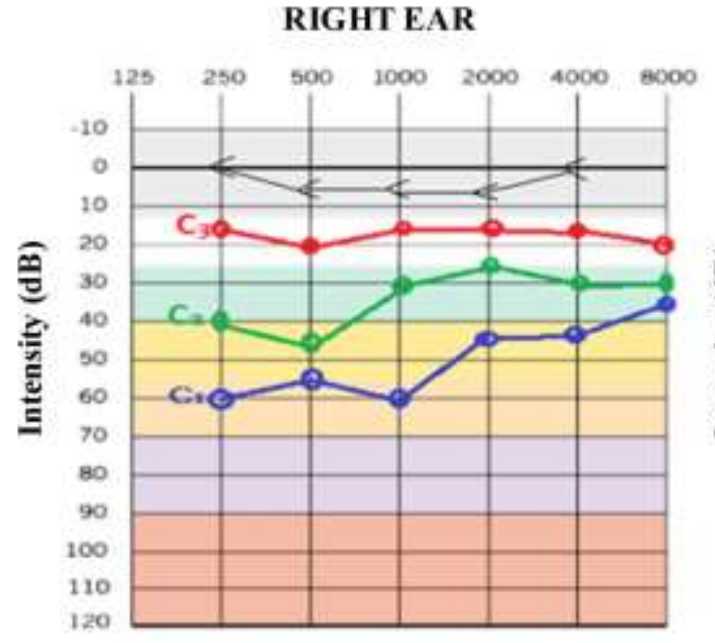

Frequency $(\mathrm{Hz})$

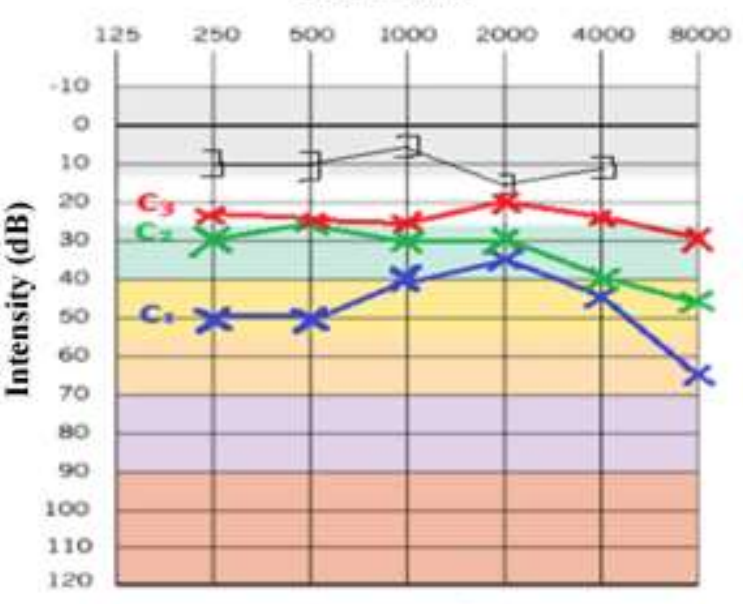

Frequency $(\mathbf{H z})$

\begin{tabular}{|l|l|l|l|l|l|l|l|l|l|}
\hline \multirow{2}{*}{ EAR } & \multicolumn{3}{|c|}{ PTA (dB) } & \multicolumn{3}{c|}{ SRT (dB) } & \multicolumn{3}{c|}{ SIS (\%) } \\
\cline { 2 - 10 } & $\mathrm{C} 1$ & $\mathrm{C} 2$ & $\mathrm{C} 3$ & $\mathrm{C} 1$ & $\mathrm{C} 2$ & $\mathrm{C} 3$ & $\mathrm{C} 1$ & $\mathrm{C} 2$ & $\mathrm{C} 3$ \\
\hline RIGHT & 52.2 & 32.5 & 12.5 & 55 & 40 & 25 & 94 & 98 & 100 \\
\hline LEFT & 42.5 & 31.2 & 10 & 55 & 35 & 20 & 96 & 98 & 100 \\
\hline
\end{tabular}

\begin{tabular}{|l|l|l|l|l|l|l|}
\hline \multirow{2}{*}{ EAR } & \multicolumn{3}{|c|}{ TYPE } & \multicolumn{3}{c|}{ Physical volume (cc) } \\
\cline { 2 - 7 } & C1 & C2 & C3 & C1 & C2 & C3 \\
\hline RIGHT & B & B & As & 0.9 & 1.1 & 1.3 \\
\hline LEFT & B & As & As & 1.3 & 1.4 & 1.2 \\
\hline
\end{tabular}

From figure 3 its evident there is an improvement in thresholds of hearing in all the frequencies across all the test conditions. The speech audiometry as well the immitance also reveals there is an improvement in the perception of the speech intelligibility and the status of the middle ear. 
Figure 4:- (a) Endoscopic view of the tympanic membrane of a patient prior to mucolite therapy (b) Endoscopic view of the tympanic membrane of a patient after mucolite therapy for three months.

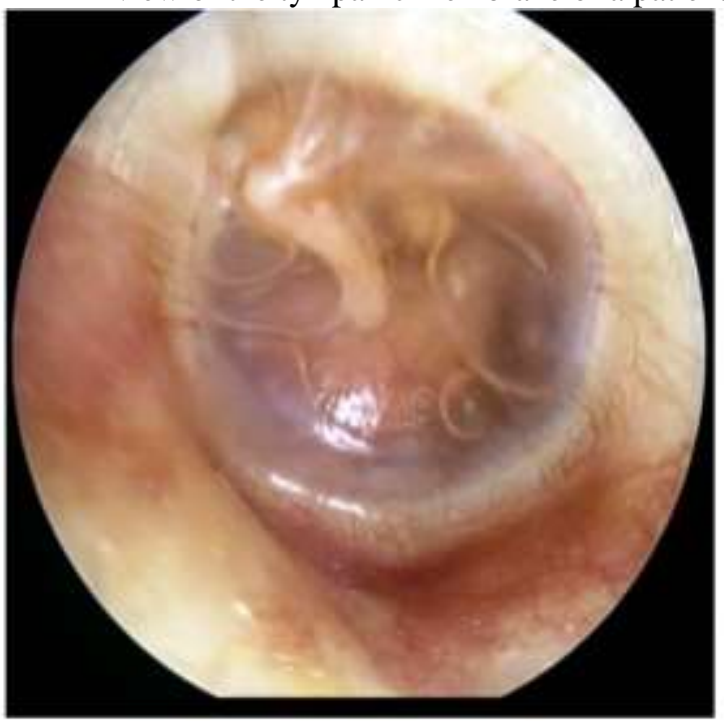

(a)

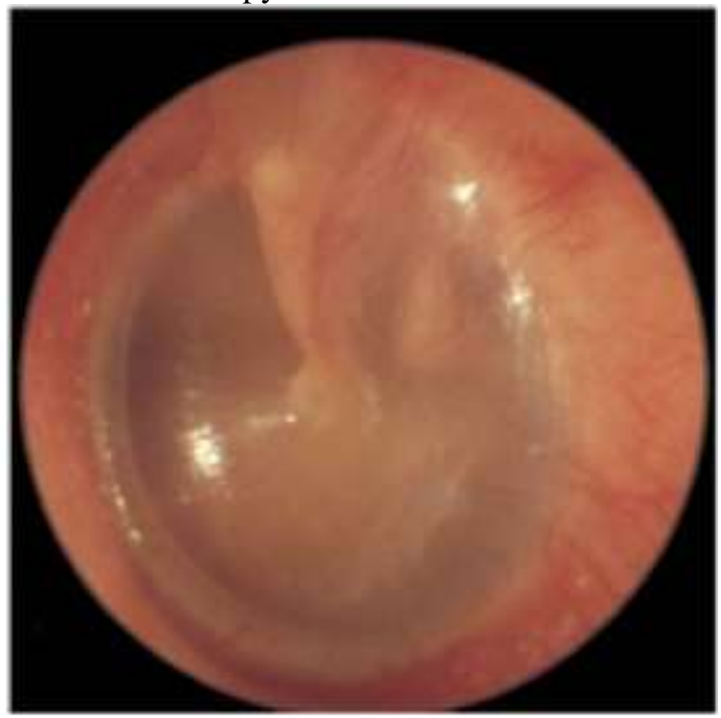

(b)

\section{Discussion:-}

Serous otitis media is the commonest cause of hearing impairment encountered by an otorhinolaryngologist. It is characterized by the accumulation of fluid in the middle ear cavity, due to alteration in the mucociliary clearance system within middle ear and Eustachian tube as a result of inadequate treatment. The main goal of treatment in serous otitis media is to restore the normal hearing and the prevention of chronic ear disease.

In our study, it was observed that the serous otitis media is common in males when compared to females, this may be due to high incidence of upper respiratory tract infections during childhood in males and less well pneumatised air cell system which may be a consequence of tubal dysfunction and more marked middle ear pathology, supporting the environmental rather than the hereditary theory of mastoid pneumatisation. It has been reported the incidence of serous otitis media in males is $63.33 \%$ and in females is $36.67 \%$ [8]. A number of medical treatment have been claimed to cure serous otitis media or to hasten its recovery. In spite of numerous claims, no convincing evidence has shown that any medical treatment is able to completely cure or to modify the natural course of the disease.

The oral steroids and antihistamines were used initially in treating SOM, thinking that it is allergic in origin, but there are no concrete evidences to support this contention because most of the patients with serous otitis media were not actually found to suffer from allergic diseases and their effusion and blood counts did not contain abnormally large number of eosinophils. The topical nasal decongestants were used in treating SOM routinely, but the fact that nasal drops hardly reach the eustachian tube [9]. So, the pharmacological effect of such preparations on ' Eustachian tube dysfunction', and their clinical efficacy has not proved the test of time [10].

Antibiotics are a useful adjunct, only if there is any features of adenoiditis, tonsillitis and sinusitis, because these conditions harbour pathogenic organisms [11]. In some cases, SOM turn or relapse into an acute otitis media and such an outcome can be prevented with the help of antibiotics. Ambroxol hydrochloride is an active N-desmethyl metabolite of bromhexine, systemically acts as an (a) mucolytic agent. It depolymerises mucopolysaccharides directly as well as by liberating lysosomal enzymes and by breaking network of fibres in thick tenacious secretions. (b) It posses surfactant property and decreases mucous viscosity by altering its structure and easy drainage of secretions through Eustachian tube. (c) It also has an anti-oxidant property.

In the present study, the data revealed that on an average the participants had threshold of mild to moderate degree of conductive hearing loss before the treatment was initiated. In Glasgow studies by Dempster and Mackenzie [12] in 1991 reported a mean of 26 decibles conductive hearing loss in serous otitis media, which is comparable to our study. After a month of follow up following treatment with mucolite (Ambroxol), the audiometric threshold become 
better with having minimal to mild conductive hearing loss. However, with regular follow up after 3 months, the thresholds were within normal limits. The speech recognition threshold (SRT) and speech identification score (SIS) also revealed a good correlation with the pure tone thresholds.

Surgical intervention is indicated only in selected resistant patients. The dilemma still continues between the choice of surgical procedure and the timing of surgery. The indication for surgical treatment is persistent effusion of longer than 3 months. In spite of benefits, placing ventilation tubes have many complications such as persistent tympanic membrane perforation, potential for introduction of a middle ear infection, early spontaneous extrusion of grommet etc. [13].

\section{Conclusion:-}

Mild to moderate type of conductive hearing loss caused by serous otitis media can be effectively treated by the mucolytic agent (Ambroxol) for long term of 1 month to 3 months duration. The other medical line of treatment used for treating SOM has higher incidence of recurrence of the disease or incomplete resolution. The prophylactic therapy of ambroxol in school going children can effectively manage the morbidity caused by serous otitis media and helps in the improvement in listening capacity and language comprehension. So, the use of ambroxol in treating serous otitis media can be considered for implementing in the National programmes like NPPCD to effectively manage the conductive deafness. However further studies are required with a large sample size.

\section{References:-}

1. Browning G. Otitis media with effusion. InScott-Brown's Otorhinolaryngology: Head and Neck Surgery 7Ed: 3 volume set 2008 Apr 25; (pp. 877-911). CRC Press.

2. Tos M. Production of mucus in the middle ear and Eustachian tube: embryology, anatomy, and pathology of the mucous glands and goblet cells in the Eustachian tube and middle ear. Annals of Otology, Rhinology \& Laryngology. 1974 Mar; 83(11_suppl):44-58.

3. Hentzer E. Ultrastructure of the middle ear mucosa. Annals of Otology, Rhinology \& Laryngology. 1976 Mar; 85(2_suppl):30-5.

4. Fiellau-Nikolajsen M, Lous J. Prospective tympanometry in 3-year-old children: a study of the spontaneous course of tympanometry types in a nonselected population. Archives of Otolaryngology. 1979 Aug 1; 105(8):461-6.

5. Tos M. Spontaneous improvement of secretory otitis and impedance screening. Archives of Otolaryngology. 1980 Jun $1 ; 106(6): 345-9$.

6. Nooni K, Sreekanth G. Otitis Media with Effusion: Comparative Effectiveness of Treatments. IOSR Journal of Dental and Medical Sciences (IOSR-JDMS) 2013;1v(15):29-34.

7. Carhart R, Jerger J. Preferred method for clinical determination of pure-tone thresholds. Journal of Speech \& Hearing Disorders. 1959.

8. American National Standards Institute (1991). Maximum Permissible Ambient Noise Levels for Audiometric Test Rooms (ANSI S3.1.1991). New York: ANSI.

9. Sade J, Luntz M, Pitashny R. Diagnosis and treatment of secretory otitis media. The otolaryngologic clinics of North America. 1989 Feb; 22(1):1-14.

10. Hoffman RA. Serous otitis media: a rationale for therapy. Bulletin of the New York Academy of Medicine. 1980 Oct; 56(8):728.

11. Ruokonen J, Sandelin K, Mäkinen J. Adenoids and otitis media with effusion. Annals of Otology, Rhinology \& Laryngology. 1979 Mar 1; 88(2):166-71.

12. Dempster JH, MacKenzie K. Tympanometry in the detection of hearing impairments associated with otitis media with effusion. Clinical otolaryngology. 1991 Apr 1; 16(2):157-9.

13. Mc Lellan C. Incidence of complications from the use of tympanostomy tubes. Arch. Otolaryngol.1980 106:9799. 